\title{
miR-708 acts as a tumor suppressor in human glioblastoma cells
}

\author{
PIN GUO $^{1}$, JIN LAN $^{1}$, JIANWEI GE $^{1}$, QUANMIN NIE $^{1}$, QING MAO $^{1,2}$ and YONGMING QIU ${ }^{1,2}$ \\ ${ }^{1}$ Department of Neurosurgery, Ren Ji Hospital, Shanghai Jiao Tong University School of Medicine, \\ Shanghai 200127; ${ }^{2}$ Shanghai Institute of Head Trauma, Shanghai 200127, P.R. China
}

Received January 25, 2013; Accepted May 3, 2013

DOI: $10.3892 /$ or.2013.2526

\begin{abstract}
Glioblastoma (GBM) is one of the most lethal forms of human cancer, and new clinical biomarkers and therapeutic targets are urgently required. microRNAs (miRNAs) are small, non-coding RNAs that negatively regulate gene expression at the post-transcriptional and/or translational level by binding the 3' untranslated regions (3' UTRs) of target mRNAs. The dysregulated expression of several miRNAs has been reported to modulate glioma progression. In the present study, we defined the expression and function of miR-708, which, based on real-time PCR analysis, were downregulated in GBM cells. The overexpression of miR-708 inhibited cell proliferation and invasion and induced apoptosis in the human GBM cell lines A172 and T98G. Furthermore, the overexpression of miR-708 reduced the expression of Akt1, CCND1, MMP2, EZH2, Parp-1 and Bcl2 in A172 and T98G cells. Taken together, our study suggests that miR-708 affects GBM cell proliferation and invasion, and induces apoptosis. It is suggested that miR-708 may play an important role as a tumor suppressor in GBM and it may be an attractive target for therapeutic intervention in GBM.
\end{abstract}

\section{Introduction}

Glioblastoma (GBM) is the highest grade glioma and is the most common malignant primary brain tumor in adults. Although there have been advances in therapy, the median survival time of patients with GBM is only 15 months (1-3). Furthermore, while our understanding of the molecular and physiological features of GBM has improved, no effective treatments for this type of cancer have been identified (4). Therefore, there is a critical need for new molecular targets,

Correspondence to: Professor Yongming Qiu or Dr Qing Mao, Department of Neurosurgery, Ren Ji Hospital, Shanghai Jiao Tong University School of Medicine, No. 1630 Dongfang Road, Pudong, Shanghai 200127, P.R. China

E-mail: qiuzhoub@hotmail.com

E-mail: maoq@netease.com

Key words: microRNA-708, proliferation, glioblastoma cells, invasion, apoptosis concepts, and approaches for treating this devastating disease. Due to the diversity and heterogeneity of GBM (5), targeting only a single factor would not result in a satisfactory clinical outcome. Thus, it would be necessary to regulate several oncogenes simultaneously.

microRNAs (miRNAs) are small, non-coding RNAs 18-24 nucleotides in length that negatively regulate gene expression at the post-transcriptional and/or translational level by binding loosely complementary sequences in the $3^{\prime}$ untranslated regions ( 3 ' UTRs) of target mRNAs (6). miRNAs regulate the expression of multiple target genes and participate in processes involved in the molecular pathology of cancer (7). Based on the concrete function and genes targeted, miRNAs can act as oncogenes or tumor suppressors $(8,9)$. One example of a tumor suppressor miRNA is miR-7, whose expression is frequently found decreased in GBM, while its overexpression reduces cell proliferation, survival and invasiveness in cultured glioma cells $(10,11)$. In contrast, miR-21 is almost invariably overexpressed in GBM and a number of other tumor types, resulting in enhanced cell motility, migration, and decreased apoptosis (12-14). miRNA profiling further reveals anomalous levels of multiple miRNAs in a wide variety of tumor types, suggesting that dysregulation of the complex network of miRNA-mRNA interactions provides growth advantages to tumor cells (15). In addition, many miRNAs have been demonstrated to function as tumor suppressors or oncogenes in glioma.

Previous studies have found that miR-708 plays different roles in several types of cancers. One example is in renal cancer cells, where miR-708 plays a role in inducing apoptosis and suppressing tumorigenicity, but in lung adenocarcinoma, increased miR-708 expression is associated with poor prognosis $(16,17)$. Therefore, the concrete function of miR-708 is associated with the cell type. Due to the inconsistent function of miR-708 in different types of cancers, studying the concrete function of miR-708 in GBM has become increasingly of interest to researchers, including ourselves. In our study, miR-708 in GBM cell lines was found to be decreased when compared with that in normal brain tissue. Therefore, we hypothesized that miR-708 may act as a tumor suppressor in GBM cell lines. In the present study, we analyzed the function of miR-708 in human glioma cell lines. We found that ectopic expression of miR-708 in A172 and T98G glioma cells caused decreased proliferation, migration and invasion, with accompanying low expression of several critical factors such as Akt1, CCND1, MMP2, EZH2, Parp-1 and Bcl2, which play 
important roles in the process of proliferation, invasion, and anti-apoptosis. Our results identify a critical role for miR-708 in the regulation of tumor progression in GBM, suggesting that miR-708 acts as a tumor suppressor gene for GBM.

\section{Materials and methods}

Tissue samples and cell culture. Human brain samples from epilepsy patients were obtained from Renji Hospital, Shanghai Jiaotong University School of Medicine, Shanghai, China. The hospital institutional review board approved this study, and written informed consent was obtained from all patients. Human A172, T98G, U87 and U251 GBM cells were obtained from the ATCC (Manassas, VA, USA). The cells were maintained in Dulbecco's modified Eagle's medium (DMEM) supplemented with $10 \%$ fetal bovine serum (FBS) (both from Gibco-BRL, Los Angeles, CA, USA) and were incubated at $37^{\circ} \mathrm{C}$ in $5 \% \mathrm{CO}_{2}$.

Plasmid construction and transfection. HEK293T cells were seeded in $60-\mathrm{mm}^{2}$ plates $\left(4 \times 10^{6}\right.$ cells/plate) in DMEM with $10 \%$ heat-inactivated FBS 1 day before transfection. Cells were transfected with $5.2 \mu \mathrm{g}$ pGIPZ-miR-708, along with $2.36 \mu \mathrm{g}$ psPAX2 and $0.8 \mu \mathrm{g}$ pMD2G plasmids using Lipofectamine 2000 in DMEM. After $6 \mathrm{~h}$, the transfection media were removed and replaced with DMEM containing $10 \%$ heat-inactivated FBS without penicillin-streptomycin. Lentiviral particles were harvested at $72 \mathrm{~h}$ post-transfection. The A172 and T98G cells were transduced with $3 \mathrm{ml}$ additional media containing viral particles and $6 \mu \mathrm{g} / \mathrm{ml}$ Polybrene. After $12 \mathrm{~h}$, the conditional media were removed and replaced with DMEM containing 10\% FBS.

To construct the miR-708 overexpression (over-miR-708) vector, 2 oligonucleotides were synthesized: forward primer, TCGAAAGGAGCTTACAATCTAGCTGGGGTGTGCTGT CCCCCAGCTAGATGTAAGCTCCTTTTTTT and reverse primer, AATTAAAAAAAGGAGCTTACAATCTAGCTGG GGGACAGCACACCCCAGCTAGATTGTAAGCTCCTT.

The oligonucleotides were annealed to be cloned into the EcoRI and XhoI sites in the pGIPZ plasmid (Open Biosystems).

Real-time PCR. RNA was extracted from tissues or cells using TRIzol (Invitrogen Life Technologies, Carlsbad, CA, USA). Real-time PCR reactions were performed with TaqMan reverse transcription reagents (Invitrogen Life Technologies) and SYBR-Green PCR Master Mix (Applied Biosystems, Foster City, CA, USA) according to the manufacturers' protocols. Normalization was performed on U6 miRNA levels. In addition, GAPDH mRNA was used as an internal control to the normalized selected genes.

Western blotting. Western blotting was performed as previously described (18), using primary antibodies against Akt1, MMP2, EZH2 (1:1,000 dilution; Santa Cruz Biotechnology, Inc., Santa Cruz, CA, USA), CCND1, GAPDH, PARP-1 and $\mathrm{Bcl} 2$ (1:1,000 dilution; Cell Signaling Technology, Inc., Danvers, MA, USA), and incubated with a horseradish peroxidase-conjugated anti-rabbit or anti-mouse antibody $(1: 1,000$ dilution, Sigma-Aldrich, St. Louis, MO, USA) for $2 \mathrm{~h}$ at room temperature.
Cell growth analysis. The cells were plated in 6-well culture plates $\left(5 \times 10^{4}\right.$ cells/well), and incubated in a humidified atmosphere of $5 \% \mathrm{CO}_{2}$ at $37^{\circ} \mathrm{C}$. Cell growth was monitored by counting cell numbers at various time intervals. Three independent experiments were carried out in triplicate.

Cell cycle analysis. The cell cycle distribution was analyzed by flow cytometry (FCM). The cells were harvested by $0.05 \%$ trypsinization, washed with phosphate-buffered saline (PBS), fixed with $75 \%$ ethanol overnight at $4^{\circ} \mathrm{C}$, and then incubated with RNase at $37^{\circ} \mathrm{C}$ for $30 \mathrm{~min}$. The nuclei were examined in a FACSCalibur flow cytometer, and DNA histograms were analyzed by Modifit software (both from Becton-Dickinson, Franklin Lakes, NJ, USA). The experiments were performed in triplicate.

Apoptosis assay. The Annexin V-FITC Apoptosis Detection Kit I (Abcam, Cambridge, MA, USA) was used to detect and qualify apoptosis by FCM according to the manufacturer's protocol. The cells were washed with cold PBS, resuspended at a density of $1 \times 10^{6}$ cells $/ \mathrm{ml}$ in $1 \mathrm{X}$ binding buffer, stained with FITC-labeled Annexin V for $5 \mathrm{~min}$, and immediately analyzed by the FACScan flow cytometer. The data were analyzed by Cell Quest software (Becton-Dickinson).

BrdU cell proliferation assay. Cell proliferation was assessed by 5-bromo-2'-deoxy-uridine (BrdU) incorporation using a Cell Proliferation ELISA, BrdU kit (Roche Applied Science, Indianapolis, IN, USA) according to the manufacturer's protocol. The cells were seeded onto a 96-well plate $\left(1 \times 10^{5}\right.$ cells/well) in $100 \mu \mathrm{l}$ culture medium and incubated at $37^{\circ} \mathrm{C}$ for $12,24,36$ and $48 \mathrm{~h}$. BrdU labeling solution was added to a final concentration of $10 \mu \mathrm{M}$, and the cells were incubated for an additional $2-4 \mathrm{~h}$ at $37^{\circ} \mathrm{C}$. The medium was removed and FixDenat (200 $\mu \mathrm{l} /$ well; Roche Applied Science) was added to the cells and incubated for $30 \mathrm{~min}$ at $25^{\circ} \mathrm{C}$. The FixDenat solution was removed completely and $100 \mu \mathrm{l} /$ well anti-BrdUPOD working solution was added and incubated for $90 \mathrm{~min}$ at $25^{\circ} \mathrm{C}$. The antibody conjugate was then removed by flicking, and the wells were washed 3 times with washing solution (200 $\mu \mathrm{l} /$ well). Substrate solution (100 $\mu \mathrm{l} /$ well) was added and the cells were incubated at $25^{\circ} \mathrm{C}$ until color development was sufficient for photometric detection (after 12, 24, 36 and $48 \mathrm{~h}$ ). The absorbance (optical density) at $450 \mathrm{~nm}$ was measured using a microplate reader.

Transwell invasion assay. The cells were detached and resuspended in serum-free medium before being plated $\left(5 \times 10^{5}\right.$ cells/well) into Matrigel-coated invasion chambers (Becton-Dickinson) and allowed to invade for $16 \mathrm{~h}$. The invading cells on the lower surface of the chambers were fixed with $70 \%$ ethanol and then stained with hematoxylin, while the remaining cells in the chambers were removed using cotton swabs. The number of remaining cells was calculated by counting five different fields under a microscope. The experiments were performed in triplicate.

Statistical analysis. GraphPad Prism 5.0 was used for statistical analysis. One-way analysis of variance (ANOVA) and Student's t-tests were used to analyze the significance between 


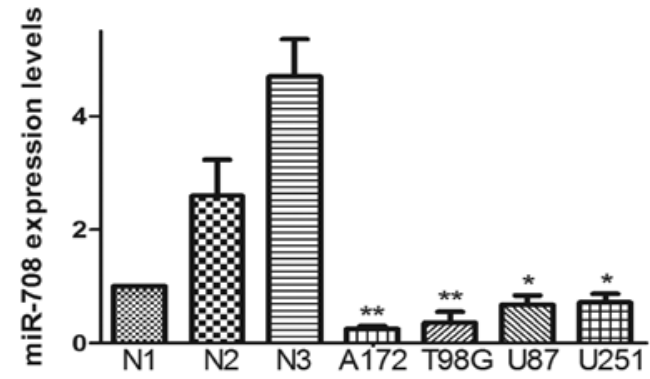

Figure 1. Expression of miR-708 in glioblastoma (GBM) cells is lower than that in normal human brain tissue (N1, N2 and N3). Total miR-708 mRNA was extracted and analyzed with real-time PCR. Data represent the means \pm SD of 3 independent experiments $\left({ }^{*} \mathrm{P}<0.05,{ }^{* *} \mathrm{P}<0.01\right)$.

groups. We considered $\mathrm{P}<0.05$ to indicate statistical significance. The means \pm standard deviation of each group were calculated for all experiments.

\section{Results}

miR-708 is downregulated in GBM cell lines. The expression of miR-708 in normal brain tissue and GBM cell lines was quantified by real-time PCR. In the present study, we extracted total RNA from the brain tissue of 2 epilepsy patients and 4 GBM cell lines (U87, U251, A172, T98G). Compared to the brain tissue, the expression of miR-708 in the 4 GBM cell lines was low (Fig. 1).

Overexpression of miR-708 inhibits GBM cell proliferation in vitro. To explore the role of miR-708 in cell proliferation, we carried out the BrdU cell proliferation and cell counting assays for 6 days. A miR-708 oligonucleotide was used to overexpress miR-708 in the A172 and T98G cells (Fig. 2). As shown in Fig. 3A and B, the overexpression of miR-708 led to poor proliferative ability in the GBM cell lines compared to the control group cells. Both the BrdU cell proliferation assay and the cell counting assay demonstrated that the overexpression of miR-708 in the A172 and T98G cells reduced their proliferative ability. The cell cycle distribution of the control and transfected cells was analyzed by FCM. In the A172 and T98G cells, miR-708 overexpression caused G0/G1 arrest (Fig. 4). These observations suggest that miR-708 overexpression suppresses the ability of GBM cells to proliferate in vitro.

miR-708 overexpression suppresses the invasive ability of GBM cells. The Transwell invasion assay was used to evaluate the impact of miR-708 expression on GBM cell invasion. Equal numbers of A172 control, A172 miR-708 overexpression cells, T98G control and T98G overexpression cells were placed in a Transwell chamber. After $16 \mathrm{~h}$, the cells that passed through the membrane were counted under a microscope. As shown in Fig. 5, the over-miR-708 cells exhibited poor invasive ability $(\mathrm{P}<0.05)$. These data suggest that miR-708 overexpression suppresses the invasive ability of GBM cells.

miR-708 overexpression induces apoptosis of GBM cells. We used FCM to assess the effect of miR-708 expression on GBM cell apoptosis. As shown in Fig. 6, statistically significant
A172

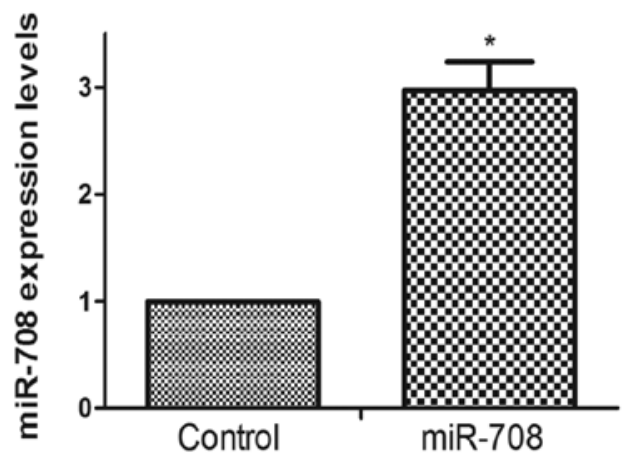

T98G

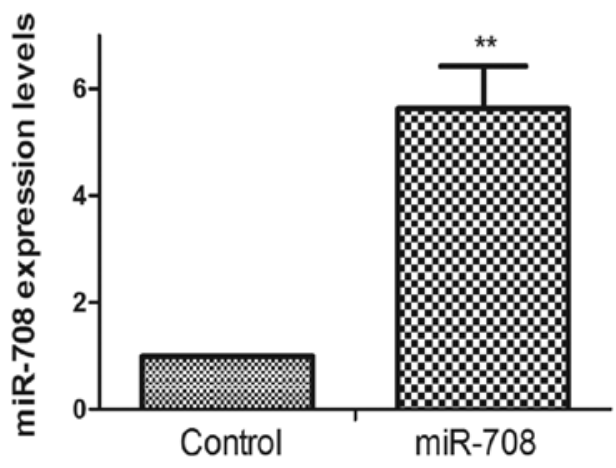

Figure 2. miR-708 mRNA upregulation following over-miR-708 oligonucleotide transfection of A172 and T98G cells. The cells were incubated with the oligonucleotide, and miR-708 mRNA was quantified by real-time PCR. Data represent the means \pm SD of 3 independent experiments. There was more miR-708 mRNA in the over-miR-708 oligonucleotide-transfected cells than in the control group $\left({ }^{*} \mathrm{P}<0.05,{ }^{* *} \mathrm{P}<0.01\right)$.

increases in Annexin $\mathrm{V}^{+}$apoptotic cells were observed in the over-miR-708 groups. This suggests that miR-708 induces GBM cell apoptosis.

miR-708 overexpression regulates the expression of components of proliferation, invasion and apoptosis factors. To investigate the potential downstream effectors of miR-708, we examined several signaling molecules that contribute to the proliferation, invasion and anti-apoptosis ability of various types of cancers. These included Akt1, CCND1, MMP2, EZH2, Parp-1 and Bcl2. For example, Akt1 and CCND1 play an important role in the proliferation of cancer cells $(19,20)$; EZH2 and MMP2 contribute to the invasion of GBM cells (21-23); and Parp-1 and Bcl2 act as anti-apoptosis factors for several types of cells $(24,25)$. As shown in Fig. 7, the expression of Akt1, CCND1, MMP2, EZH2, Parp-1 and $\mathrm{Bcl} 2$ in the over-miR-708 cells was reduced when compared to the levels in the control group cells.

\section{Discussion}

In the present study, we identified an important tumorsuppressive miRNA, miR-708, that plays a critical role in the process of GBM. Compared to the brain tissue from epilepsy patients, the GBM cell lines A172, T98G, U87 and U251 
A

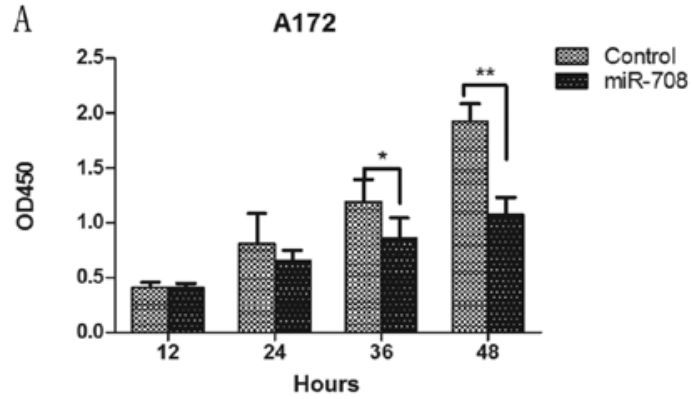

B

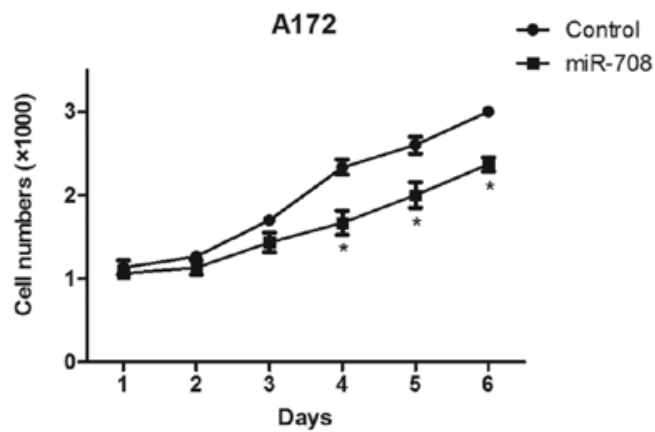

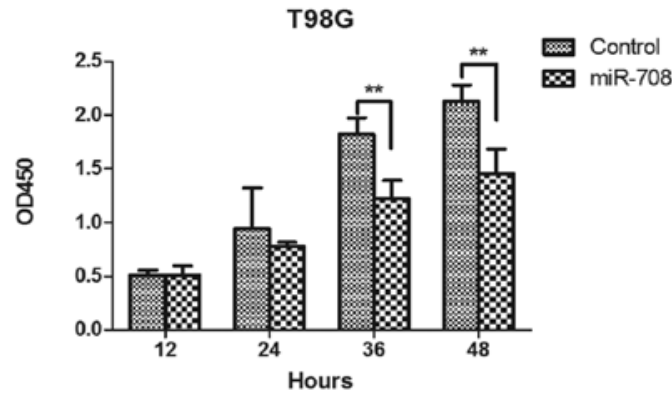

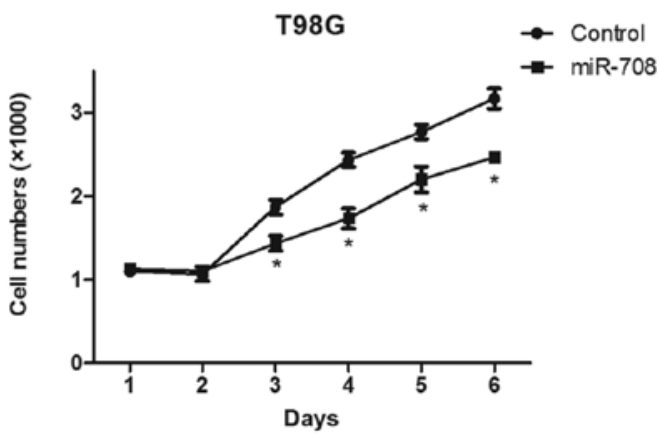

Figure 3. miR-708 inhibits the proliferative ability of glioblastoma (GBM) cells. (A) The proliferative ability of the cells was compared at separate times using a BrdU proliferation assay. (B) Equal numbers of control and over-miR-708 cells were incubated, and were counted on separate days. Data represent the means \pm SD of 3 independent experiments $\left({ }^{*} \mathrm{P}<0.05,{ }^{* *} \mathrm{P}<0.01\right)$.
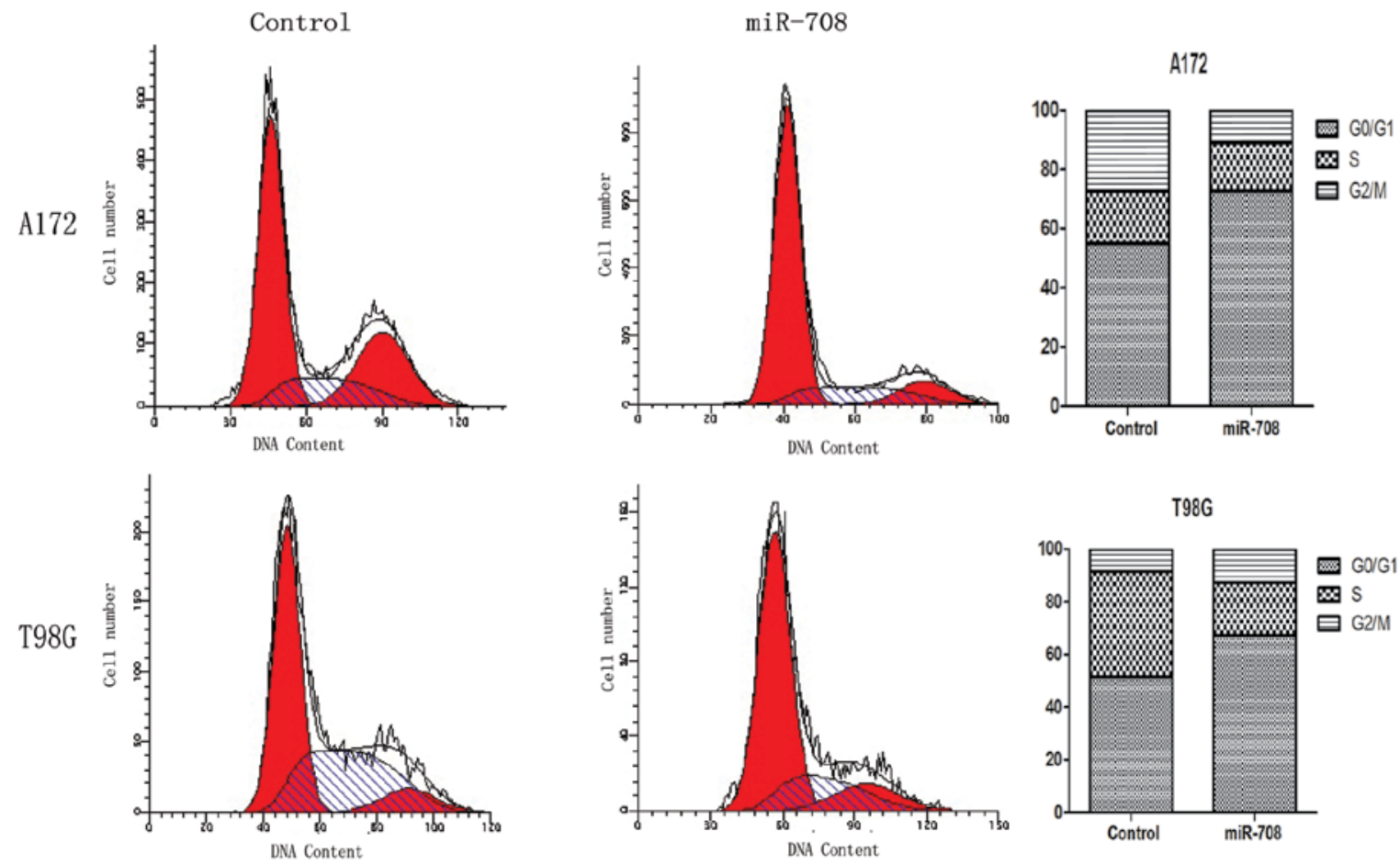

Figure 4. miR-708 overexpression induces G0/G1 arrest and inhibits cell growth. Cell counting was performed by flow cytometry (FCM). The percentages of cells in the G0/G1, S, and G2/M phase are indicated. FCM data, represented as histograms, revealed a significant increase in the percentage of cells in the $\mathrm{G} 0 / \mathrm{G} 1$ phase in all groups of cells.

contained low amounts of miR-708. The ectopic expression of miR-708 decreased the proliferation and invasion of GBM cells and induced apoptosis in the cells. In other words, the expression level of miR-708 may negatively regulate glioma malignancy. These data suggest that miR-708 plays a critical role in the glioma malignant phenotype and it may function as an antitumor factor in glioma cells. Several studies have reported on the dysregulation of various miRNAs in GBM, 

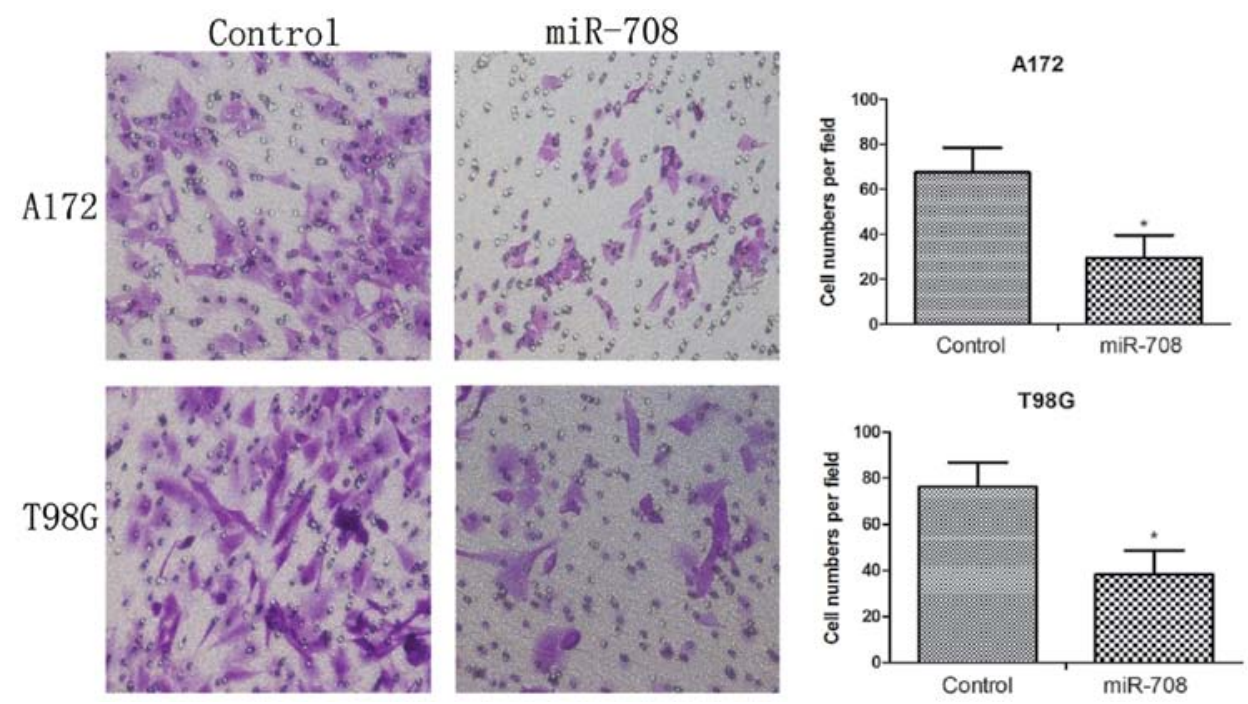

Figure 5. miR-708 reduces the invasive ability of glioblastoma (GBM) cells. The Transwell assay showed that the control A172 and T98G cells exhibited stronger invasive ability than the over-miR-708 groups. Data represent the means \pm SD of 3 independent experiments ( $(\mathrm{P}<0.05)$.
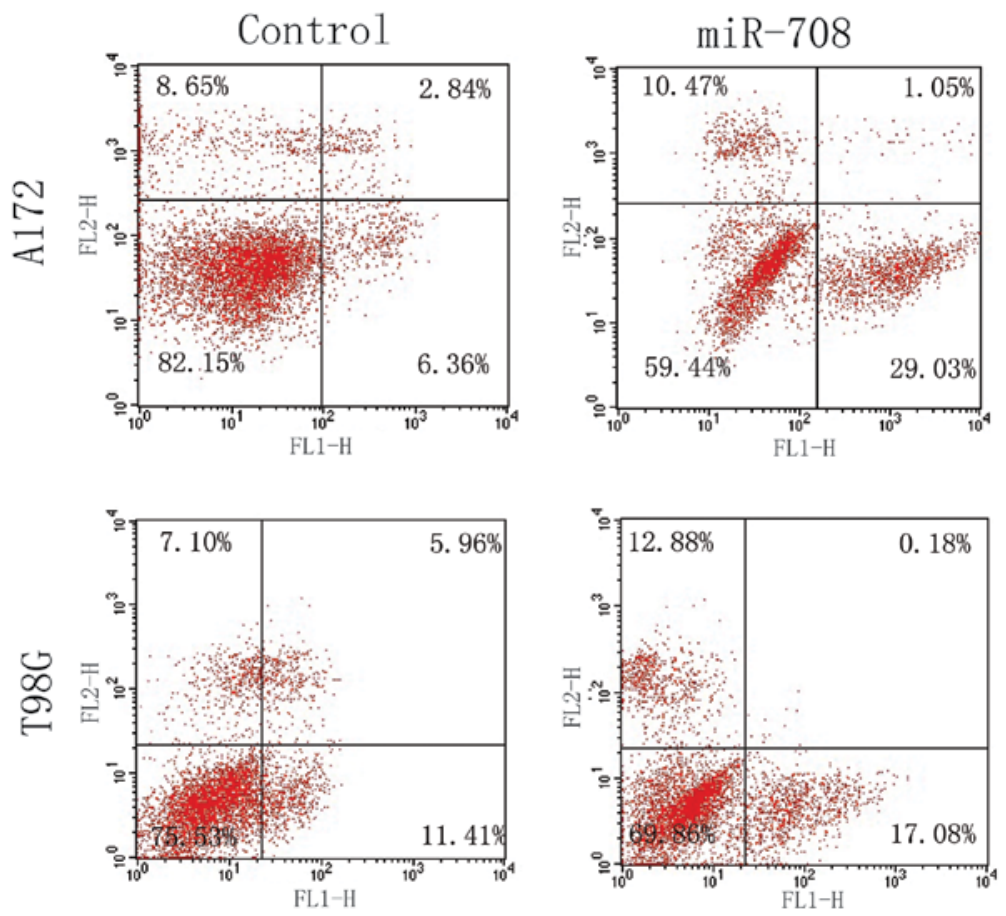

Figure 6. miR-708 induces apoptosis. A172 and T98G cells were transfected with over-miR-708 oligonucleotides. All the cells were harvested, stained for Annexin $\mathrm{V}$ and propidium iodide (PI), and analyzed by flow cytometry (FCM). Early apoptotic cells were Annexin $\mathrm{V}^{+} / \mathrm{PI}^{+}$, necrotic cells were Annexin $\mathrm{V}^{-} / \mathrm{PI}^{+}$, and healthy cells were Annexin $\mathrm{V}^{-} / \mathrm{PI}^{-}$. The figure depicts a representative experiment.

such as let-7, miR-21, miR-221/222 and miR-10b (11,13,26-28). However, this is the first report that implicates miR-708 in the process of GBM.

Our studies indicate that miR-708 modulates the expression profiles of multiple genes involved in cell proliferation, invasion and apoptosis. Moreover, these genes are aberrantly expressed in cancer. Akt1 and CCND1 play a critical role in the regulation of cell proliferation, particularly Akt1, which can increase cell proliferation through the Akt1 pathway $(19,20)$. CCND1 can also affect cell proliferation through regulation of the cell cycle $(29,30)$. Dysregulation of CCND1 can also be correlated with the aggressive behavior of a tumor. Lebeau et al (30) reported that CCND1 regulates glioma cell proliferation by controlling progression to the G1 phase of the cell cycle. In addition, our data showed that miR-708 overexpression induced G0/G1 arrest, subsequently reducing the proliferative ability of GBM cells. The effects of Akt1 and CCND1 provide strong evidence for miR-708 as a tumor suppressor that regulates cell proliferation in GBM cells.

Targeting the invasive ability of cells is an important treatment strategy for different types of tumors (31), including GBM. Aberrant expression of many genes, which act as 


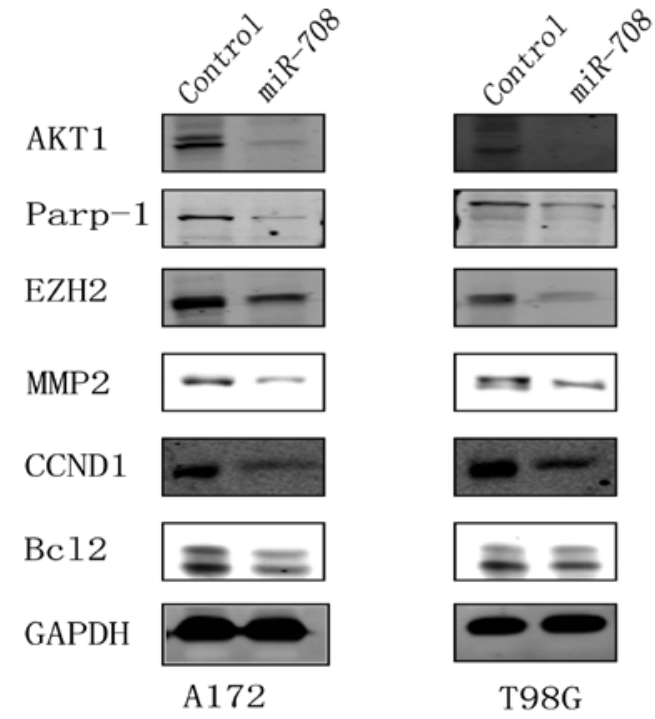

Figure 7. miR-708 regulates factors that play a role in the proliferation, invasion and anti-apoptosis of glioblastoma (GBM) cells. When miR-708 was overexpressed in the A172 and T98G cells, the factors AKT1, Parp-1, EZH2, MMP2, CCND1 and Bcl2 were reduced.

promoters of cell invasion, are observed in cancer. MMP2 is one of the key enzymes involved in the degradation of the extracellular matrix, and its high expression is associated with tumor cell invasion $(23,32)$. In addition, EZH2 plays a role in increasing the invasive ability of several tumor cell types $(33,34)$. Our data showed that MMP2 and EZH2 were reduced when miR-708 was overexpressed in GBM cells. The regulation of MMP2 and EZH2 also provides support for miR-708 as a tumor suppressor that targets cell invasion.

Apoptosis is a well-orchestrated cellular mechanism that balances cell proliferation and cell death (35). The ability to evade apoptosis is a hallmark of tumorigenesis. As prior studies have demonstrated that Parp-1 and $\mathrm{Bcl} 2$ are antiapoptotic proteins, the increased expression of these genes could contribute to tumor progression $(24,25)$. In our study, the expression of Parp-1 and Bcl 2 in the over-miR-708 GBM cells was low in contrast to the control group cells. These data add additional credibility to the tumor suppressor role of miR-708 in GBM.

In addition, Saini et al (16) reported that miR-708 plays a tumor-suppressive role in renal cancer cells, while Jang et al (17) confirmed that miR-708 acts as an oncogene contributing to tumor growth and disease progression by directly downregulating TMEM88 in lung cancer. This phenomenon is plausible because of the biodiversity of tumors, indicating that the concrete function of miR-708 depends on the cell type.

In conclusion, we demonstrated that the overexpression of miR-708 in the human GBM cell lines A172 and T98G significantly affected cell proliferation, invasion and apoptosis. Our data suggest that these biological effects may be related to the modulated expression of Akt1, CCND1, MMP2, EZH2, Parp-1 and Bcl2. However, the exact mechanism of miR-708 regulation in these genes requires further study. The effect of GBM cell proliferation, invasion, and apoptosis by miR-708 suggests that it may act as a tumor suppressor in human GBM.
Furthermore, miR-708 may be an attractive target for therapeutic intervention in GBM.

\section{Acknowledgements}

This study was supported by a project for building hospital clinical key disciplines (no. RJ.4101307) and a grant from the Shanghai government (no. 0952nm03900).

\section{References}

1. Stupp R, Mason WP, van den Bent MJ, et al: Radiotherapy plus concomitant and adjuvant temozolomide for glioblastoma. N Engl J Med 352: 987-996, 2005.

2. Chin L and Gray JW: Translating insights from the cancer genome into clinical practice. Nature 452: 553-563, 2008.

3. Wiedemeyer R, Brennan C, Heffernan TP, et al: Feedback circuit among INK4 tumor suppressors constrains human glioblastoma development. Cancer Cell 13: 355-364, 2008.

4. Dunn GP, Rinne ML, Wykosky J, et al: Emerging insights into the molecular and cellular basis of glioblastoma. Genes Dev 26: 756-784, 2012.

5. Bonavia R, Inda MM, Cavenee WK and Furnari FB: Heterogeneity maintenance in glioblastoma: a social network. Cancer Res 71: 4055-4060, 2011.

6. Bartel DP: MicroRNAs: genomics, biogenesis, mechanism, and function. Cell 116: 281-297, 2004.

7. Sassen S, Miska EA and Caldas C: MicroRNA: implications for cancer. Virchows Arch 452: 1-10, 2008.

8. Shenouda SK and Alahari SK: MicroRNA function in cancer: oncogene or a tumor suppressor? Cancer Metastasis Rev 28: 369-378, 2009

9. Chen CZ: MicroRNAs as oncogenes and tumor suppressors. N Engl J Med 353: 1768-1771, 2005.

10. Lee ST, Chu K, Oh HJ, et al: Let-7 microRNA inhibits the proliferation of human glioblastoma cells. J Neurooncol 102: 19-24, 2011.

11. Kefas B, Godlewski J, Comeau L, et al: microRNA-7 inhibits the epidermal growth factor receptor and the Akt pathway and is down-regulated in glioblastoma. Cancer Res 68: 3566-3572, 2008.

12. Papagiannakopoulos T, Shapiro A and Kosik KS: MicroRNA-21 targets a network of key tumor-suppressive pathways in glioblastoma cells. Cancer Res 68: 8164-8172, 2008.

13. Chan JA, Krichevsky AM and Kosik KS: MicroRNA-21 is an antiapoptotic factor in human glioblastoma cells. Cancer Res 65: 6029-6033, 2005.

14. Gabriely G, Wurdinger T, Kesari S, et al: MicroRNA 21 promotes glioma invasion by targeting matrix metalloproteinase regulators. Mol Cell Biol 28: 5369-5380, 2008.

15. Lu J, Getz G, Miska EA, et al: MicroRNA expression profiles classify human cancers. Nature 435: 834-838, 2005.

16. Saini S, Yamamura S, Majid S, et al: MicroRNA-708 induces apoptosis and suppresses tumorigenicity in renal cancer cells. Cancer Res 71: 6208-6219, 2011.

17. Jang JS, Jeon HS, Sun Z, et al: Increased miR-708 expression in NSCLC and its association with poor survival in lung adenocarcinoma from never smokers. Clin Cancer Res 18: 3658-3667, 2012.

18. Su CH, Zhao R, Zhang F, et al: 14-3-3sigma exerts tumorsuppressor activity mediated by regulation of COP1 stability. Cancer Res 71: 884-894, 2011.

19. Vivanco I and Sawyers CL: The phosphatidylinositol 3-kinase AKT pathway in human cancer. Nat Rev Cancer 2: 489-501, 2002.

20. Sommer G, Dittmann J, Kuehnert J, et al: The RNA-binding protein La contributes to cell proliferation and CCND1 expression. Oncogene 30: 434-444, 2011.

21. Suvà ML, Riggi N, Janiszewska M, et al: EZH2 is essential for glioblastoma cancer stem cell maintenance. Cancer Res 69: 9211-9218, 2009.

22. Orzan F, Pellegatta S, Poliani PL, et al: Enhancer of Zeste 2 $(\mathrm{EZH} 2)$ is up-regulated in malignant gliomas and in glioma stem-like cells. Neuropathol Appl Neurobiol 37: 381-394, 2011.

23. Luo Y, Liang F and Zhang ZY: PRL1 promotes cell migration and invasion by increasing MMP2 and MMP9 expression through Src and ERK1/2 pathways. Biochemistry 48: 1838-1846, 2009. 
24. Sugo N, Niimi N, Aratani Y, Masutani M, Suzuki H and Koyama H: Decreased PARP-1 levels accelerate embryonic lethality but attenuate neuronal apoptosis in DNA polymerase $\beta$-deficient mice. Biochem Biophys Res Commun 354: 656-661, 2007.

25. Gross A, McDonnell JM and Korsmeyer SJ: BCL-2 family members and the mitochondria in apoptosis. Genes Dev 13: 1899-1911, 1999

26. Zhang C, Zhang J,Hao J, et al: High level of miR-221/222 confers increased cell invasion and poor prognosis in glioma. J Transl Med 10: 119, 2012.

27. Zhang J, Han L, Ge Y, et al: miR-221/222 promote malignant progression of glioma through activation of the Akt pathway. Int J Oncol 36: 913-920, 2010.

28. Guessous F, Alvarado-Velez M, Marcinkiewicz L, et al: Oncogenic effects of miR-10b in glioblastoma stem cells. J Neurooncol 112: 153-163, 2013.

29. Jain M, Kumar S, Upadhyay R, et al: Influence of apoptosis (BCL2, FAS), cell cycle (CCND1) and growth factor (EGF, EGFR) genetic polymorphisms on survival outcome: an exploratory study in squamous cell esophageal cancer. Cancer Biol Ther 6: 1553-1558, 2007.
30. Lebeau A, Unholzer A, Amann G, et al: EGFR, HER-2/neu, cyclin D1, p21 and p53 in correlation to cell proliferation and steroid hormone receptor status in ductal carcinoma in situ of the breast. Breast Cancer Res Treat 79: 187-198, 2003.

31. Livant DL: Targeting invasion induction as a therapeutic strategy for the treatment of cancer. Curr Cancer Drug Targets 5: 489-503, 2005.

32. Westermarck J and Kähäri VM: Regulation of matrix metalloproteinase expression in tumor invasion. FASEB J 13: 781-792, 1999.

33. Ren G, Baritaki S, Marathe H, et al: Polycomb protein EZH2 regulates tumor invasion via the transcriptional repression of the metastasis suppressor RKIP in breast and prostate cancer. Cancer Res 72: 3091-3104, 2012.

34. Rao ZY, Cai MY, Yang GF, et al: EZH2 supports ovarian carcinoma cell invasion and/or metastasis via regulation of TGF- $\beta 1$ and is a predictor of outcome in ovarian carcinoma patients. Carcinogenesis 31: 1576-1583, 2010.

35. Subramanian S and Steer CJ: MicroRNAs as gatekeepers of apoptosis. J Cell Physiol 223: 289-298, 2010. 